Case Report

\title{
Assessing Biological Response to Bevacizumab Using 18F-Fluoromisonidazole PET/MR Imaging in a Patient with Recurrent Anaplastic Astrocytoma
}

\author{
Ramon F. Barajas Jr., ${ }^{1}$ Miguel H. Pampaloni, ${ }^{1}$ Jennifer L. Clarke, ${ }^{2}$ Youngho Seo, ${ }^{1,3}$ \\ Dragana Savic, ${ }^{1}$ Randall A. Hawkins, ${ }^{1}$ Spencer C. Behr, ${ }^{1}$ Susan M. Chang, ${ }^{2}$ Mitchel Berger, ${ }^{2}$ \\ William P. Dillon, ${ }^{1}$ and Soonmee $\mathrm{Cha}^{1,2}$ \\ ${ }^{1}$ Department of Radiology and Biomedical Imaging, University of California, San Francisco, 505 Parnassus Avenue, \\ M-391, San Francisco, CA 94143-0628, USA \\ ${ }^{2}$ Department of Neurological Surgery, University of California, San Francisco, 505 Parnassus Avenue, Room 779 M, \\ San Francisco, CA 94143-0112, USA \\ ${ }^{3}$ Department of Radiation Oncology, UCSF Long Hospital, 505 Parnassus Avenue, San Francisco, CA 94143-0226, USA
}

Correspondence should be addressed to Soonmee Cha; soonmee.cha@ucsf.edu

Received 22 December 2014; Revised 19 January 2015; Accepted 2 February 2015

Academic Editor: Marco Leonardi

Copyright (C) 2015 Ramon F. Barajas Jr. et al. This is an open access article distributed under the Creative Commons Attribution License, which permits unrestricted use, distribution, and reproduction in any medium, provided the original work is properly cited.

We present our initial experience in using single modality fluoromisonidazole (FMISO) PET/MR imaging to noninvasively evaluate the biological effects induced by bevacizumab therapy in a patient treated for recurrent high grade glioma. In this index patient, bevacizumab therapy resulted in the development of nonenhancing tumor characterized by reduced diffusion and markedly decreased FMISO uptake in the setting of maintained CBF and CBV. These observations suggest that the dynamic biological interplay between tissue hypoxia and vascular normalization occurring within treated recurrent high grade glioma can be captured utilizing FMISO PET/MR imaging.

\section{Introduction}

Glioma is the most common type of primary intra-axial brain tumor. Despite the aggressive combination of surgery, irradiation, and temozolomide based chemotherapy, median overall survival remains less than 15 months for high grade glioma [1]. Tumor recurrence remains the primary cause of mortality in this patient population with treatment options becoming limited and mostly ineffective when conventional therapy fails.

One of the pathologic hallmarks of malignant glioma recurrence is the presence of hypoxia. Hypoxia is a potent stimulator of vascular endothelial growth factor (VEGF) mediated angiogenesis [2]. In addition, hypoxia limits the efficacy of radiation therapy and chemotherapy. A better understanding of treatment related changes in regional tumor hypoxia may allow for the development of more effective patient-specific therapies. To this end, angiogenesis inhibitor therapy is currently administered to patients who have developed glioma recurrence. Bevacizumab, one of the most widely used angiogenesis inhibitors for glioma recurrence, is a recombinant humanized monoclonal antibody against VEGF. The goal of antiangiogenesis therapy is to disrupt and normalize neoplastic vascular formation in an attempt to prevent further tumor growth. Given the complex and synergistic interplay between hypoxia and angiogenesis, the pretherapeutic quantification of hypoxic tumor burden may be useful in risk stratification of patients who seek to undergo angiogenesis inhibitor therapy.

One noninvasive means of quantifying tissue hypoxia is through the use of $18 \mathrm{~F}$-fluoromisonidazole (FMISO) PET imaging. Several studies have validated FMISO uptake as a robust measure of tissue hypoxia [2-8]. Reduced preradiation hypoxic volume, as measured by FMISO PET, has been 
shown to be a good prognostic biomarker of clinical outcome in patients with treatment naïve glioblastoma [3]. Physiologic MR imaging methods such as dynamic susceptibilityweighted (DSC) perfusion and diffusion-weighted imaging (DWI) sequences may also contribute to the characterization of tumor biology and prognosis for patients with recurrent glioma. Combined, these PET/MR imaging sequences provide an opportunity to study the biological association among tumor hypoxia (by FMISO PET and DWI), cellularity (by DWI), and microvascularity (by DSC perfusion MRI). The combination of imaging data would be expected to be synergistic in delineating the mechanism between hypoxia and angiogenesis. As such, the assessment of hypoxic tumor burden prior to angiogenesis inhibitor therapy may provide useful information in predicting response for patients with recurrent glioma. However, there is currently a paucity of evidence regarding the role of hypoxic tumor burden in imaging response outcome following bevacizumab therapy.

In this case report we describe the FMISO PET/MR imaging changes that occurred in a patient with recurrent anaplastic astrocytoma undergoing bevacizumab therapy. Specifically, we demonstrate that, in this patient, bevacizumab therapy results in the progressive growth of recurrent nonenhancing cellular tumor manifested by reduced diffusion with decreased tumor hypoxia.

\section{Case Presentation}

A 65-year-old man with a history of left temporal lobe WHO grade III anaplastic astrocytoma presented with clinical and MR imaging evidence of tumor recurrence 9 months after initial diagnosis. At the time of diagnosis the tumor had molecular features consisting of isocitrate dehydrogenase 1 (IDH-1) wild type, epidermal growth factor receptor (EGFR) amplified, and O6-methylguanin-DNAmethyltransferase (MGMT) unmethylated. Following subtotal resection, the patient underwent external beam radiation therapy (6,000 cGy; 30 fractions) and concurrent temozolomide therapy for 8 months. Upon the diagnosis of glioma recurrence, the patient was enrolled in a clinical trial that included a bevacizumab treatment arm $(10 \mathrm{mg} / \mathrm{kg}, 879 \mathrm{mg}$ IV every two weeks).

One week prior to initiation of bevacizumab and following the fourth dose of therapy the patient underwent Institutional Review Board approved FMISO PET/MR imaging. Following informed written consent, the patient was administered $7 \mathrm{mCi}$ FMISO intravenously. Simultaneous PET/MR imaging was performed on a 3T investigational general electric (GE) scanner 90 minutes following FMISO administration. Forty-minute PET emission imaging was performed with time of flight reconstruction. Attenuation correction utilized patient-specific T1-weighted map registered with segmented bone from a head CT image template. $\mathrm{MR}$ imaging sequences included an axial $\mathrm{T} 1$ precontrast (567/5 msec, TR/TE; 5/0 0 Slice Thickness/Skip (mm)), 3D CUBE FLAIR (5.34/163/2375 msec, TR/TE/TI; $1 / 0 \mathrm{~mm}), 3 \mathrm{D}$ T2 (3000/90 msec; $1 / 0 \mathrm{~mm})$, axial diffusion weighted imaging (DWI) $\left(8000 / 5 \mathrm{msec} ; 2 / 0 \mathrm{~mm} ; 1,000 \mathrm{sec} / \mathrm{mm}^{2}\right.$ B-value), dynamic susceptibility weighted contrast enhanced (DSC) perfusion imaging (1400/25 $\mathrm{msec} ; 3 / 0 \mathrm{~mm} ; 35^{\circ}$ flip angle), and $3 \mathrm{D}$ gradient recalled T1-weighted postcontrast $(34 / 3 \mathrm{msec}$; $1 / 0 \mathrm{~mm}$ ) imaging.

Processing of DSC and DWI data was performed using a GE advantage workstation running Functool software v4.4. This allowed for the production of cerebral blood volume $(\mathrm{CBV})$, cerebral blood flow $(\mathrm{CBF})$, and apparent diffusion coefficient (ADC) physiologic maps. FMISO PET and MR sequences were coregistered using investigational GE PET/MR review v1.0 allowing for the production of regions of interest (ROIs) and generation of quantitative values. DSC and DWI maps were standardized to contralateral normal appearing white matter (NAWM) allowing for the production of relative values. Semiquantitative FMISO values were produced by standardization to ROIs placed on the right distal cervical internal carotid artery (tissue to blood; T/B value) and NAWM (rValue).

Pre-bevacizumab FMISO PET/MR imaging demonstrated contrast enhancing recurrent disease about the posterior margin of the resection cavity associated with elevated $\mathrm{CBV}\left(\mathrm{CBV}_{\text {min }} 10.3, \mathrm{CBV}_{\text {mean }}\right.$ 59.2, and $\mathrm{CBV}_{\text {max }}$ 154.8; $\mathrm{rCBV}_{\text {min }} 1.25, \mathrm{rCBV}_{\text {mean }} 1.84$, and $\left.\mathrm{rCBV}_{\max } 2.17\right)$ and $\mathrm{CBF}$ $\left(\mathrm{CBF}_{\text {min }} 76.5, \mathrm{CBF}_{\text {mean }} 397.2\right.$, and $\mathrm{CBF}_{\text {max }} 773.8 ; \mathrm{rCBF}_{\text {min }}$ 1.55, $\mathrm{rCBF}_{\text {mean }} 1.23$, and $\left.\mathrm{rCBV}_{\max } 1.24\right)$. FMISO uptake within the contrast enhancing lesion was also elevated (T/B values, min 1.12 , mean 1.43, and max 1.65; rValues, min 1.72, mean 2.11, and $\max 2.18)$ without associated reduced diffusion $\left(\mathrm{ADC}_{\min } 935\right.$, $\mathrm{ADC}_{\text {mean }} 1500$, and $\mathrm{ADC}_{\max } 2690 ; \mathrm{rADC}_{\text {min }} 1.50, \mathrm{rADC}_{\text {mean }}$ 1.86, and $\mathrm{rADC}_{\max }$ 2.53) (Figure 1(a)). FMISO PET/MR imaging 10 weeks after the initial exam ( 8 weeks following first dose of bevacizumab therapy, 4 doses administered as total) demonstrated a nonenhancing T2/FLAIR hyperintense mass about the posterior margin of the resection cavity that had developed reduced diffusion $\left(\mathrm{ADC}_{\min } 534, \mathrm{ADC}_{\text {mean }} 707\right.$, and $\mathrm{ADC}_{\max } 1370 ; \mathrm{rADC}_{\min } 0.77, \mathrm{rADC}_{\text {mean }} 0.81$, and $\mathrm{rADC}_{\max }$ 1.24) and decreased FMISO uptake (T/B values: $\min 0.46$, mean 0.91, and max 1.43; rValues: min 0.49, mean 0.71, and $\max 1.65$ ) (Figure 1(b)). The nonenhancing reduced diffusion lesion maintained $\mathrm{CBV}\left(\mathrm{CBV}_{\min } 8.09, \mathrm{CBV}_{\text {mean }} 50.8\right.$, and $\mathrm{CBV}_{\text {max }} 190.6 ; \mathrm{rCBV}_{\text {min }} 1.09, \mathrm{rCBV}_{\text {mean }} 0.99$, and $\mathrm{rCBV}_{\text {max }}$ 0.99) and $\mathrm{CBF}\left(\mathrm{CBF}_{\min } 61.3, \mathrm{CBF}_{\text {mean }} 295.4\right.$, and $\mathrm{CBF}_{\max }$ $761.5 ; \mathrm{rCBF}_{\text {min }} 1.28 \mathrm{rCBF}_{\text {mean }} 1.18$, and $\left.\mathrm{rCFV}_{\text {max }} 1.44\right)$. To date the patient continues bevacizumab therapy.

\section{Discussion}

We highlight our initial experience utilizing single modality FMISO PET/MR imaging to noninvasively evaluate the biological effects induced by bevacizumab therapy in patients treated for recurrent high grade glioma. In this index patient, bevacizumab therapy resulted in the development of nonenhancing tumor characterized by reduced diffusion and markedly decreased FMISO uptake in the setting of maintained $\mathrm{CBF}$ and CBV. These observations suggest that the dynamic biological interplay between tumor hypoxia and vascular normalization occurring within a patient with recurrent high grade glioma treated with bevacizumab can be captured utilizing FMISO PET/MR imaging. 


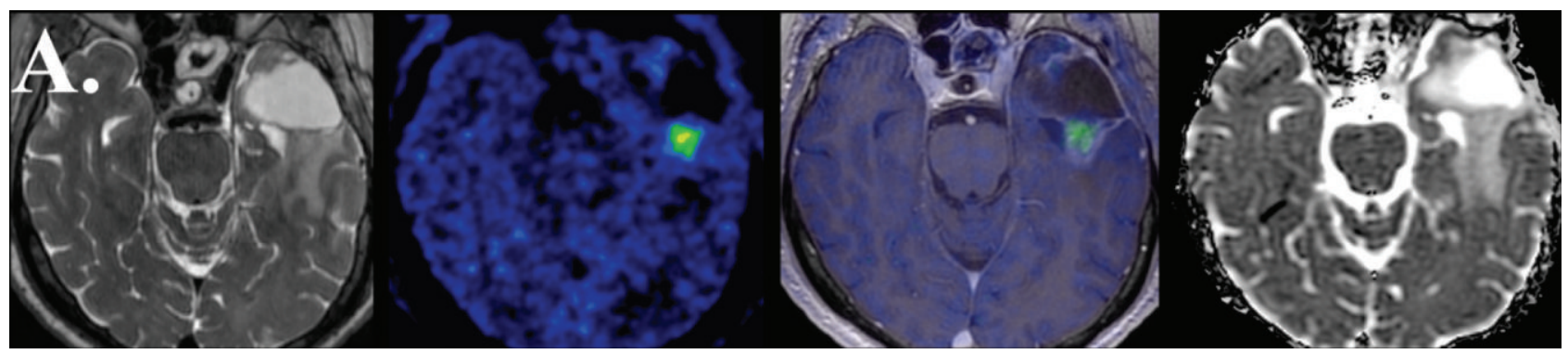

(a)

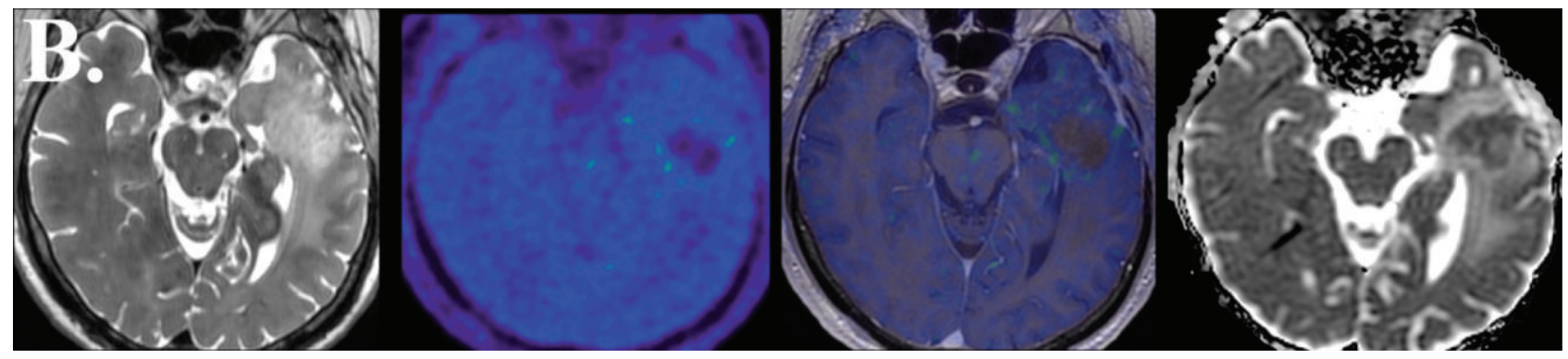

(b)

FIGURE 1: Single modality FMISO PET/MR imaging in a 65-year-old man with recurrent left temporal lobe WHO grade III anaplastic astrocytoma immediately prior to (a) and concurrent with bevacizumab therapy (b). Baseline, simultaneously obtained, axial T2 weighted (left), FMISO PET (middle left), fused FMISO PET and T1 weighted post contrast (middle right), and apparent diffusion coefficient (right) $\mathrm{PET} / \mathrm{MR}$ imaging demonstrate recurrence of disease evidenced by contrast enhancing focus bordering the posterior margin of an anterior left temporal lobe resection cavity. This region demonstrates increased FMISO uptake with no evidence of associated reduced diffusion. Followup PET/MR imaging 10 weeks later (after 4 doses of bevacizumab) demonstrates the development of a nonenhancing T2 hyperintense mass about the posterior margin of the resection cavity that is associated with the development of reduced diffusion and diminished FMISO uptake.

The development of reduced diffusion concurrent with bevacizumab therapy is a well-established MR imaging feature, the etiology of which remains unclear [9-11]. Some investigations suggest that these lesions reflect aggressive hypercellular tumor growth. Other studies suggest that these lesions represent chronic hypoxia and necrotic tissue. In our patient, the region of reduced diffusion maintained baseline perfusion levels; however, it demonstrated FMISO uptake below the levels observed within normal appearing white matter. This finding seemed paradoxical as reduced diffusion would be expected in the setting of tissue hypoxia. In this case, we hypothesize that the emergence of a nonenhancing mass characterized by reduced diffusion and decreased FMISO uptake concurrent with bevacizumab therapy represents the presence of highly cellular recurrent glioma with transformed or normalized tumor microvasculature $[12,13]$.

The development of lesions with reduced diffusion concurrent with bevacizumab therapy has been shown to be prognostic of improved clinical outcomes [14]. The antagonism of VEGF receptors decreases the expression of abnormal microvascular morphology. This therapeutic effect is postulated to improve transport of oxygen and drug therapies [15]. As a result, one would presume to find improved efficacy of radiation therapy in patients manifesting reduced diffusion associated with decreased hypoxic burden following bevacizumab administration. It is important to highlight the point that DWI alone would not allow for the differentiation of hypoxic tissue from cellular recurrent tumor. Therefore, the combined examination of FMISO and DWI metrics in this patient population may allow for lesion biological stratification prior to the initiation of radiation therapy.

FMISO uptake has been previously characterized within newly diagnosed and recurrent glioma; however, to our knowledge, this is the first report of FMISO PET/MR imaging following bevacizumab therapy in humans with recurrent glioma. Cher et al. have previously investigated the aggressive biological features associated with FMISO uptake in 14 patients with treatment of naïve glioma [8]. Their investigation demonstrated a statistically significant association among FMISO uptake, vascular endothelial growth factor, and Ki-67 immunoreactivity suggesting that glioma hypoxia is biologically associated with angiogenesis and tumor cellular proliferation prior to therapeutic intervention. The association between aggressive biological features and FMISO uptake is consistent with the observations made in our patient's pretherapeutic PET/MR examination at the time of tumor recurrence: elevated FMISO uptake within the contrast enhancing region of recurrent tumor with elevated cerebral blood volume.

It is yet to be determined whether the loss of contrast enhancement within recurrent glioma following bevacizumab therapy should be considered a treatment success. According to the current response assessment in neurooncology (RANO) criteria the loss of contrast enhancement 
would be considered a complete response to therapy [16]. However, the clinical significance of this finding has yet to be definitively adjudicated. Furthermore, the development of reduced diffusion lesions following bevacizumab therapy, according to some investigations, would suggest the development of recurrent nonenhancing disease. The results of our study preliminarily indicate that these nonenhancing reduced diffusion lesions that develop following bevacizumab therapy are not the result of tissue hypoxia. Continued investigation into the prognostic utility of FMISO uptake following prolonged bevacizumab therapy still needs to be undertaken.

\section{Conclusion}

Our initial experience suggests that FMISO PET/MR imaging can noninvasively evaluate the dynamic effects induced by bevacizumab therapy in a patient with recurrent high grade glioma. We continue to enroll patients in this study to further adjudicate the biological effects of bevacizumab therapy on tumor hypoxic burden.

\section{Conflict of Interests}

The authors declare that there is no conflict of interests regarding the publication of this paper.

\section{Acknowledgments}

The first author thanks Bethany Barajas for her helpful comments regarding this paper, Vahid Ravanfar for his helpfulness in the imaging of our patient, and Sharmila Majumdar Ph.D. for the opportunity to undertake this research endeavor. This work was supported by Grant no. [5T32EB001631-07] from the National Institutes of Health and the University of California San Francisco, Department of Radiology and Biomedical Imaging.

\section{References}

[1] R. Stupp, W. P. Mason, M. J. van den Bent et al., "Radiotherapy plus concomitant and adjuvant temozolomide for glioblastoma," The New England Journal of Medicine, vol. 352, no. 10, pp. 987-996, 2005.

[2] M. W. Dewhirst, Y. Cao, and B. Moeller, "Cycling hypoxia and free radicals regulate angiogenesis and radiotherapy response," Nature Reviews Cancer, vol. 8, no. 6, pp. 425-437, 2008.

[3] A. M. Spence, M. Muzi, K. R. Swanson et al., "Regional hypoxia in glioblastoma multiforme quantified with $\left[{ }^{18} \mathrm{~F}\right] \mathrm{flu}-$ oromisonidazole positron emission tomography before radiotherapy: correlation with time to progression and survival," Clinical Cancer Research, vol. 14, no. 9, pp. 2623-2630, 2008.

[4] M. Bruehlmeier, U. Roelcke, P. A. Schubiger, and S. M. Ametamey, "Assessment of hypoxia and perfusion in human brain tumors using PET with $18 \mathrm{~F}$-fluoromisonidazole and ${ }^{15} \mathrm{O}-$ $\mathrm{H}_{2} \mathrm{O}$," Journal of Nuclear Medicine, vol. 45, no. 11, pp. 1851-1859, 2004.

[5] H. J. Tochon-Danguy, J. I. Sachinidis, F. Chan et al., "Imaging and quantitation of the hypoxic cell fraction of viable tumor in an animal model of intracerebral high grade glioma using [18F]fluoromisonidazole (FMISO)," Nuclear Medicine and Biology, vol. 29, no. 2, pp. 191-197, 2002.

[6] P. E. Valk, C. A. Mathis, M. D. Prados, J. C. Gilbert, and T. F. Budinger, "Hypoxia in human gliomas: demonstration by PET with fluorine-18- fluoromisonidazole," Journal of Nuclear Medicine, vol. 33, no. 12, pp. 2133-2137, 1992.

[7] M. Zimny, B. Gagel, E. DiMartino et al., "FDG-a marker of tumour hypoxia? A comparison with $\left[{ }^{18} \mathrm{~F}\right]$ fluoromisonidazole and $\mathrm{pO}_{2}$-polarography in metastatic head and neck cancer," European Journal of Nuclear Medicine and Molecular Imaging, vol. 33, no. 12, pp. 1426-1431, 2006.

[8] L. M. Cher, C. Murone, N. Lawrentschuk et al., "Correlation of hypoxic cell fraction and angiogenesis with glucose metabolic rate in gliomas using $18 \mathrm{~F}$-fluoromisonidazole, $18 \mathrm{~F}$ FDG PET, and immunohistochemical studies," Journal of Nuclear Medicine, vol. 47, no. 3, pp. 410-418, 2006.

[9] J. Rieger, O. Bähr, K. Müller, K. Franz, J. Steinbach, and E. Hattingen, "Bevacizumab-induced diffusion-restricted lesions in malignant glioma patients," Journal of Neuro-Oncology, vol. 99, no. 1, pp. 49-56, 2010.

[10] E. R. Gerstner, M. P. Frosch, and T. T. Batchelor, "Diffusion magnetic resonance imaging detects pathologically confirmed, nonenhancing tumor progression in a patient with recurrent glioblastoma receiving bevacizumab," Journal of Clinical Oncology, vol. 28, no. 6, pp. e91-e93, 2010.

[11] E. R. Gerstner, M. P. Frosch, and T. T. Batchelor, "Diffusion magnetic resonance imaging detects pathologically confirmed, nonenhancing tumor progression in a patient with recurrent glioblastoma receiving bevacizumab," Journal of Clinical Oncology, vol. 28, no. 6, pp. e91-e93, 2010.

[12] R. F. Barajas Jr., J. L. Rubenstein, J. S. Chang, J. Hwang, and S. Cha, "Diffusion-weighted MR imaging derived apparent diffusion coefficient is predictive of clinical outcome in primary central nervous system lymphoma," American Journal of Neuroradiology, vol. 31, no. 1, pp. 60-66, 2010.

[13] T. Sugahara, Y. Korogi, M. Kochi et al., "Usefulness of diffusionweighted MRI with echo-planar technique in the evaluation of cellularity in gliomas," Journal of Magnetic Resonance Imaging, vol. 9, no. 1, pp. 53-60, 1999.

[14] S. Mong, B. M. Ellingson, P. L. Nghiemphu et al., "Persistent diffusion-restricted lesions in bevacizumab-treated malignant gliomas are associated with improved survival compared with matched controls," The American Journal of Neuroradiology, vol. 33, no. 9, pp. 1763-1770, 2012.

[15] R. K. Jain, "Delivery of molecular medicine to solid tumors: lessons from in vivo imaging of gene expression and function," Journal of Controlled Release, vol. 74, no. 1-3, pp. 7-25, 2001.

[16] P. Y. Wen, D. R. Macdonald, D. A. Reardon et al., "Updated response assessment criteria for high-grade gliomas: response assessment in neuro-oncology working group," Journal of Clinical Oncology, vol. 28, no. 11, pp. 1963-1972, 2010. 


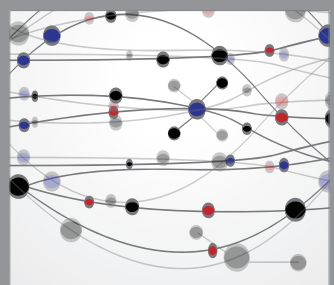

The Scientific World Journal
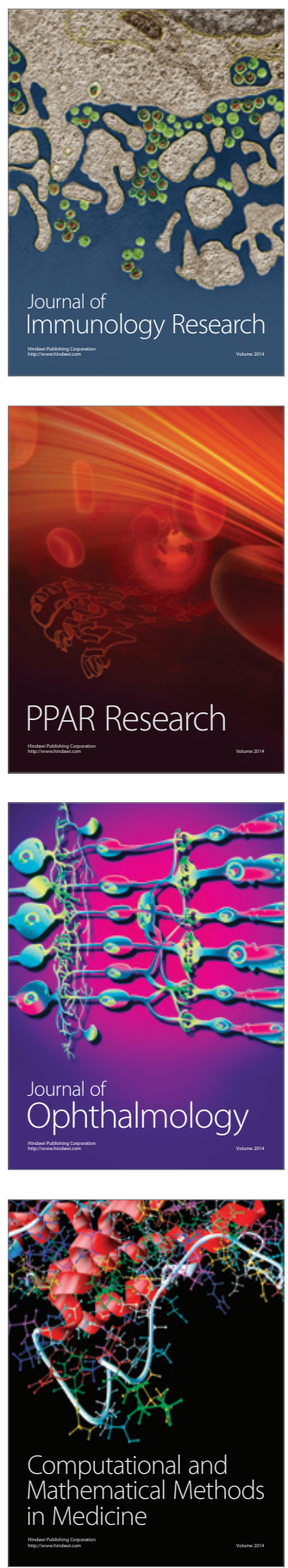

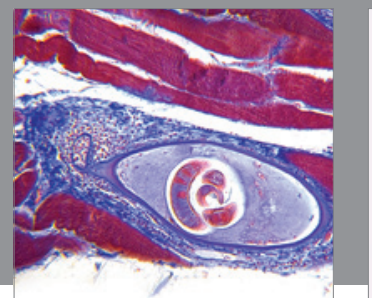

Gastroenterology

Research and Practice
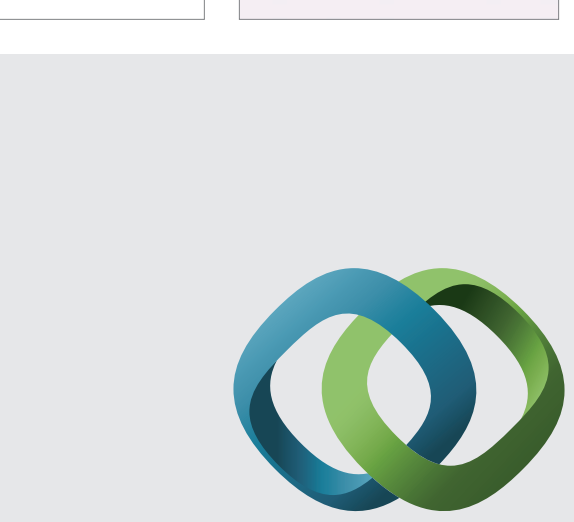

\section{Hindawi}

Submit your manuscripts at

http://www.hindawi.com
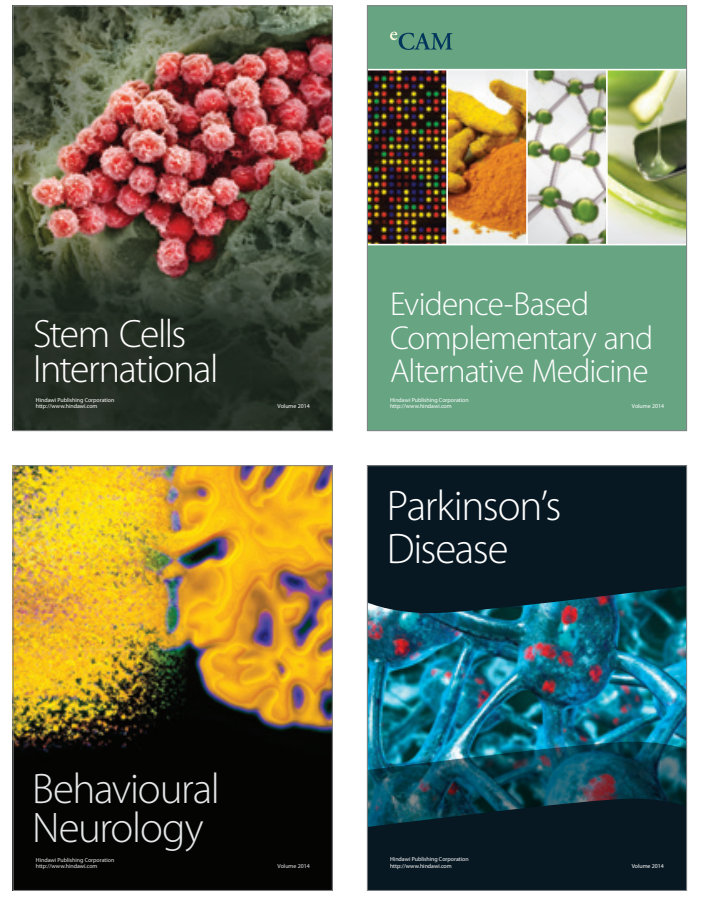
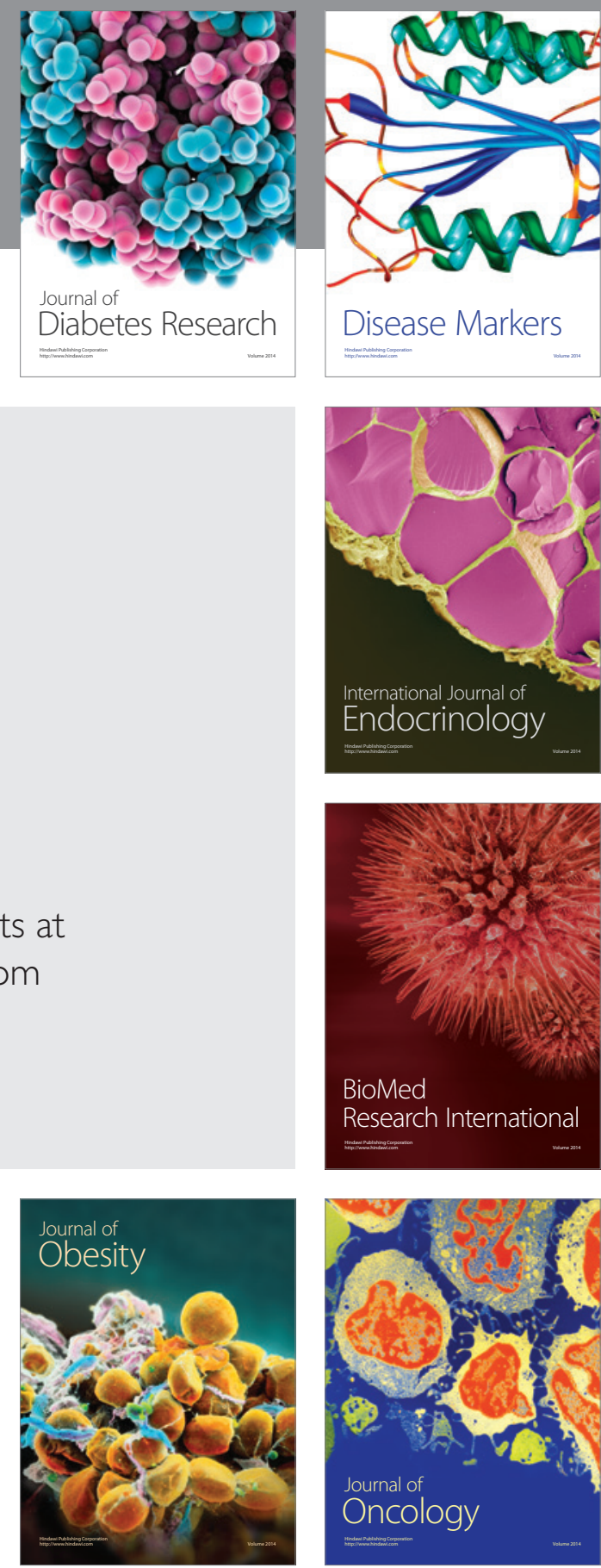

Disease Markers
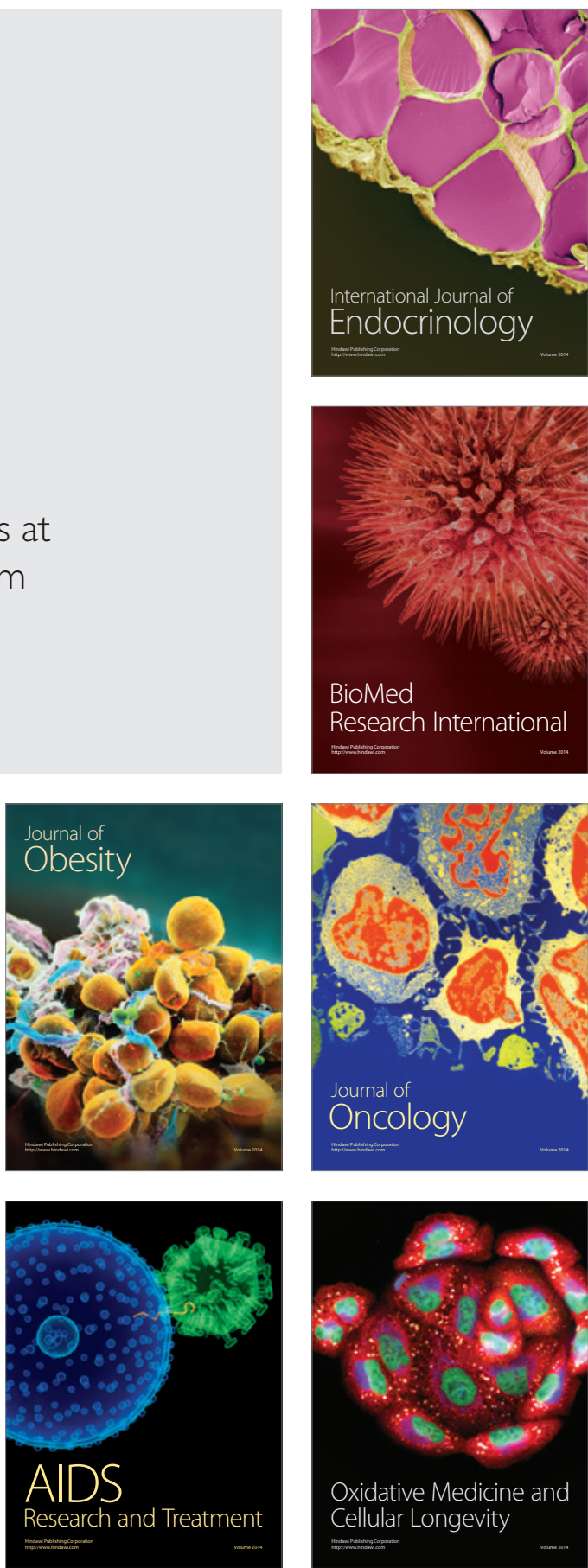